\section{Bipolare Störungen Breiter Therapieansatz vorteillhaft}

- Der Anteil manischer Patienten, die mithilfe von Antipsychotika eine dauerhafte Remission von mindestens einem Jahr erreichen, sei mit unter $20 \%$ deutlich geringer als allgemein angenommen, mahnte Professor Ana Gonzales-Pinto, Vitoria/Spanien, auf dem 28. CINP-Kongress. Faktoranalysen hätten gezeigt, dass auch subklinische depressive Symptome das Outcome manischer Episoden negativ beeinflussen. Die strenge kategoriale Einteilung bipolarer Störungen werde daher den therapeutischen Erfordernissen im klinischen Alltag nicht gerecht, bestätigte Professor Steven Potkin, Irvine/USA. Die Behandlung müsse breiter ansetzen und beispielsweise auch kognitive Defizite berücksichtigen. Sie seien wichtige Prädiktoren für Krankheitseinsicht und Therapietreue sowie den langfristigen Funktionsstatus.

Das Antipsychotikum Asenapin (Sycrest ${ }^{\circledR}$ ), zugelassen für die Behandlung mäßiger bis schwerer Episoden einer Bipolar-I-Störung, biete mit seinem einzigartigen Rezeptorprofil interessante therapeutische Ansatzpunkte für die große Bandbreite der klinischen Symptome, meinte Professor Frank Tarazi, Boston/USA. Anders als bei anderen Antipsychotika komme es unter Asenapin zu einer Hochregulation am Dopamin $\mathrm{D}_{1}$-Rezeptor, die mit einer kognitiven Verbesserung verknüpft sei. Eine weitere Besonderheit sei die Hochregulation der serotonergen $5-\mathrm{HT}_{1 \mathrm{a}}$-Rezeptoren im Hippocampus, der eine Verringerung der emotionalen Dysregulation zugeschrieben werde.

In der Klinik wichtig sei der rasche Wirkbeginn bereits ab dem zweiten Tag, denn eine schnelle Response korreliere positiv mit einer langfristigen Stabilisierung, sagte Professor Heinz Grunze, Newcastle/UK. Unter Asenapin verzeichneten in den Studien auch weniger Patienten nach drei Monaten eine Zunahme depressiver Symptome als unter dem Vergleichsantipsychotikum Olanzapin. Für Grunze besitzt Asenapin prinzipiell wichtige Eigenschaften zum Einsatz als Stimmungsstabilisierer. Allerdings gebe es hierfür noch nicht genügend klinische Daten.

Dr. Alexander Kretzschmar

Satellitensymposium „Asenapine - a multifonctional antipsychotic. Beyond symptom control in bipolar disorder" im Rahmen des 28. World Congress of Neuropsychopharmakologie des Collegium Internationale Neuropsychiatricum (CINP), Stockholm, 4.6.2012; Veranstalter: Lundbeck

\title{
Tierexperimentelle Untersuchung
}

\section{Ginkgo kann Hirn und Gedächtnis vor Hypoxieschåden schüitzen}

- Kann ein Ginkgo biloba-Extrakt (EGb761) dank antioxidativer und neuroprotektiver Eigenschaften Patienten mit Schlafapnoe vor Hirnschäden durch Sauerstoffmangel schützen und Gedächtnisdefizite verhüten? Tierversuche eines ägyptisch-arabischen Wissenschaftlerteams sprechen dafür.

Untersucht wurde die Hypothese in einer Versuchsreihe mit 80 Ratten. 21 Tage lang wurden die Tiere in-

\section{Hans-Jörg Weitbrecht Wissenschaftspreis}

Zum dreizehnten Mal wird der mit $10.000 €$ dotierte Preis für besondere Leistungen auf dem Gebiet der klinischen Neurowissenschaften von Bayer HealthCare Deutschland ausgeschrieben. Für den Preis können sich Autoren bewerben oder vorgeschlagen werden, die herausragende Arbeiten zur Ursachen- und /oder Therapieforschung bei neurologischen und/oder psychiatrischen Erkrankungen geleistet haben und nicht älter als 45 Jahre sind. Interessenten senden ihre Arbeiten (bis zu drei thematisch zusammenhängende Arbeiten), die nicht älter als fünf Jahre alt und zur Publikation akzeptiert oder publiziert sein sollten, bis zum 1. März 2013 in deutscher oder englischer Sprache mit einseitiger Zusammenfassung, Bibliografie und Lebenslauf an: Universitätsklinikum Düsseldorf, Neurologische Klinik, Professor H.-P. Hartung, Moorenstr. 5, 40225 Düsseldorf. Weitere Informationen bei thomas.glaser@bayer.com. termittierender Hypoxie unterschiedlicher Stärke ausgesetzt. Der Effekt von EGb761 in verschiedenen Dosierungen ( 50 und $100 \mathrm{mg} / \mathrm{kg} \mathrm{KG}$ ) wurde mittels PARTest (Passive Avoidance Reflex Test) sowie anhand verschiedener biochemischer Parameter überprüft.

Unter dem Einfluss des langfristigen Sauerstoffmangels zeigten sich bei den Tieren deutliche Gedächtnisstörungen, dokumentiert durch eine signifikante $\mathrm{Ab}$ nahme der Retentionslatenz im PAR-Test. Begleitet wurde die kognitive Beeinträchtigung durch Anzeichen von oxidativem Stress und stressinduzierte neuronale DNS-Schäden in für kognitive Funktionen relevanten Bereichen des Hippocampus.

EGb761 bewirkte eine signifikante Umkehrung beziehungsweise Abschwächung dieser Prozesse. Gedächtnisdefizite wurden aufgehoben, Markersubstanzen wie MDA (Malondialdehyd) und NO in Serum und Hippocampus signifikant reduziert, antioxidative Abwehrmechanismen reaktiviert. Nach Ansicht der Autoren zeigen diese Befunde, dass EGb761 in der Lage ist, nicht nur zentral, sondern auch in peripheren Geweben vor oxidativen DNS-Schäden zu schützen. Ursula Einecke 